\title{
Ruedas de investigación narrativa en la formación de profesores de química
}

\section{Narrative research wheels in the training of chemistry teachers}

\author{
Aline Machado Dorneles, ${ }^{*}$ Maria do Carmo Galiazzi**
}

Recibido: 7 de junio de 2020 Aceptado: 22 de septiembre de 2020 Publicado: 30 de septiembre de 2020

To cite this article: Dorneles, A. y Galazzi, M. (2020). Ruedas de investigación narrativa en la formación de profesores de química. Márgenes, Revista de Educación de la Universidad de Málaga, 1 (3), 344-354

DOI: https://doi.org/10.24310/mgnmar.v1i3.9499

\section{RESUMEN}

Este artículo busca comprender el papel de la experiencia narrativa en la construcción de la identidad profesional del docente de Química. También propone la investigación narrativa como una forma de construir conocimiento en Ciencias Naturales. Para ello, se presenta un estudio con enfoque fenomenológico-hermenéutico, que permitió trazar un camino metodológico y, por tanto, interpretar las narrativas. Se entiende que las experiencias documentadas en forma narrativa mejoran los procesos colaborativos de conversación, lectura, escritura y reescritura en la formación de los docentes. Por tanto, las interpretaciones construidas llevan a comprender la experiencia narrada y a argumentar que la escritura narrativa proporciona una forma de pensar y hablar sobre la formación del profesorado en Química. En este sentido, se discute la importancia de las Ruedas de Investigación Narrativa en la formación de los profesores de Química como una posibilidad para construir conocimientos, compartir experiencias y, así, favorecer el aprendizaje y apropiación del lenguaje narrativa en Química.

Palabras clave: investigación investigación narrativa; ruedas de formación; profesorado en química

\section{ABSTRACT}

This article seeks to understand the role of the narrative experience in the construction of the professional identity of the Chemistry teacher. It also proposes narrative research as a way to build knowledge in Natural Sciences. To do this, a study with a phenomenological-hermeneutical approach is presented, which allowed us to trace a methodological path and, therefore, interpret the narratives. It is understood that the experiences documented in narrative form improve the collaborative processes of conversation, reading, writing and rewriting in the training of teachers. Therefore, the constructed interpretations lead us to understand the narrated experience and to argue that narrative writing provides a way of thinking and talking about teacher training in Chemistry. In this sense, the importance of Narrative Research Wheels in the training of Chemistry teachers is discussed as a possibility to build knowledge, share experiences and, thus, favor the learning and appropriation of narrative language in Chemistry.

Keywords: narrative research; training wheels; teacher in chemistry

*Aline Machado Dorneles, 0000-0001-7110-9378

Universidade Federal de Río Grande (Brasil)

alinedorneles@furg.br

\footnotetext{
**Maria do Carmo Galiazzi, 0000-0003-0513-0018

Universidade Federal de Río Grande (Brasil) mariagaliazzi@furg.br mcgaliazzi@gmail.com
} 


\section{INTRODUCCIÓN}

En este artículo presentamos el proceso de investigación narrativa en la formación de profesores de Química, como una forma de construir conocimiento a través de experiencias y conocimientos compartidos en círculos de capacitación (DORNELES, 2016). Decidimos recorrer los caminos de la investigación narrativa porque entendemos que la investigación no se realiza sola, sino con la presencia del otro, con las historias que nos convierten en maestros e investigadores de nuestra propia práctica educativa. De esta manera, entendemos que las experiencias cuando se documentan en forma narrativa mejoran los procesos de colaboración de conversación, lectura, escritura y reescritura en la formación de docentes (Suárez, 2011).

El texto está organizado en tres momentos. Primero, presentamos el lugar de la investigación, las ruedas de investigación narrativa desarrolladas en la componente curricular de prácticas pedagógicas supervisadas II del curso de Química en la Universidad Federal de Río Grande (FURG)-Brasil. Los encuentros semanales estuvieron mediados por la escritura narrativa, como una forma de favorecer la comprensión de los conocimientos vividos al realizar la experimentación en la enseñanza de la Química.

En segundo lugar, las contribuciones teóricas y metodológicas de la investigación narrativa permiten construir entendimientos sobre la experiencia narrada. (Clandinin; Connelly, 2011). El estudio del enfoque fenomenológico-hermenéutico permitió trazar una historia metodológica y, por lo tanto, interpretar y comprender cada historia narrada. Las narrativas que constituyeron este trabajo son formas de decir acerca de la experiencia, y fueron expresadas por un conjunto de mónadas, que son formas de construir sentido sobre lo narrado, y así convertirse en narrativas comunicables y experimentables (Rosa et al, 2011).

Concluimos con la presentación de la experiencia narrativa presente en cada mónada narrada por los maestros del componente curricular de prácticas pedagógicas supervisadas II. Construimos entendimientos sobre la experiencia narrativa en la formación de profesores de Química, a partir de la historia construida en el curso de Licenciatura en Química en FURG en el que la experiencia narrativa estaba presente, con la idea de proponer autoría, invención e investigación en rueda.

\section{LAS RUEDAS DE INVESTIGACIÓN NARRATIVA: CONTEXTO DE EXPERIENCIA DOCENTE}

La investigación narrativa aquí presentada propone una formación horizontal entre profesores y académicos de Química, con la oportunidad de vivir experiencias. A partir de esto, nos preguntamos: ¿cómo nos convertimos en sujetos de la experiencia en el proceso de pensar y hablar sobre las prácticas educativas en la enseñanza de la Química?

En las ruedas de investigación narrativa en el curso de Química, específicamente en el componente curricular de prácticas pedagógicas supervisadas II, es para la mayoría de los estudiantes la primera experiencia de enseñanza. Así se propone la planificación de prácticas experimentales como una forma de investigar fenómenos químicos. Las actividades son una invitación a vivir una experiencia docente y, de este modo, promover la investigación narrativa en la construcción del conocimiento químico y pedagógico. 
En las reuniones semanales de la disciplina buscamos crear ruedas de afecto y aprendizaje sobre la experimentación y la formación de profesores de Química. Las ruedas hay un encuentro con el otro, un intercambio de palabras, pensamientos, historias y sentimientos. Vivimos la experiencia del encuentro, nos abrimos a la posibilidad de vivir nuevas experiencias, de ser sensibles a ella. Necesitamos aprender a silenciar todo el discurso teórico, suposiciones, explicaciones, necesitamos de palabras que nos ayuden a escuchar lo vivido de una manera diferente, más atentos a lo que hay. Para que la experiencia de la relación con otro nos llegue, no desde nuestras categorías, sino en el ejercicio de deshacerlas (Contreras, 2014).

Proponemos la investigación narrativa en rueda como una forma de entender la experiencia (Clandinin; Connelly, 2011). La investigación desde la perspectiva de la experiencia pone en juego el proceso de pensamiento, porque si pensamos que es porque algo se nos ocurre, nos pasa e nos afecta. Y también inventamos, porque el pensamiento tiene que inventar, para decir desde otro plano lo que sucede solo cuando vivimos. La invención permite pensar de forma no repetitiva y codificada (Contreras, 2014).

La idea es concebir la experimentación en química como un acontecimiento. El acontecimiento entendido como el lugar de donde provienen las preguntas. Por lo tanto, se propuso una clase que pregunta qué sucedió (Geraldi, 2010). "La atención al acontecimiento es la atención al ser humano y su complejidad. Tomar la clase como un evento es elegir el flujo del movimiento como inspiración, rechazando la permanencia del mismo y la morbosa fijación del pasado"( Geraldi, 2010, p.100).

Para esto, era importante abrir la posibilidad de inventar, crear y repensar las actividades experimentales. Mediante la construcción de preguntas como estrategia inicial de investigación. Entendemos que las preguntas permiten construir un objeto que se puede perfeccionar, como un artefacto de conocimiento que se perfecciona en el trabajo colaborativo entre los participantes (Wells, 2001). El proceso de investigación narrativa constituye cuestionar, repensar y escribir narrativamente en rueda, proporcionando así oportunidades para el aprendizaje y la construcción de conocimiento químico y pedagógico en la formación de profesores de Química.

\section{CAMINO METODOLÓGICO EN LA INVESTIGACIÓN NARRATIVA}

No puedes explicar una historia; Todo lo que puedes hacer es darle varias interpretaciones.

Jerome Bruner (2001)

Como investigadores narrativos, nos damos cuenta de que no hay una única forma de transformar los textos de campo en textos de investigación. La atención se centra en las experiencias vividas por los sujetos. De esta forma, presentamos cómo llegamos a los textos de investigación, el proceso de interpretación de las narrativas, en la construcción de entendimientos sobre la experiencia narrada. El estudio del análisis fenomenológico fue importante para que pudiera componer un movimiento de persecución al interrogatorio y, por lo tanto, no tomar los datos descritos como pragmáticos cuyos significados ya estarían contenidos allí, pero pasa por las decisiones en 
la búsqueda para conocer las formas de proceder a rendir cuentas del demandado. Entonces, la pregunta de esta investigación es: ¿qué es lo que muestra al desarrollar la investigación narrativa de la experimentación en la formación de profesores de Química?

A continuación, describimos los primeros pasos del camino para interpretar el fenómeno de la experimentación en la formación de profesores de Química, basado en los estudios de Bicudo (2011); Martins, Bicudo (1989). Señalo aproximaciones de fenomenología con la investigación narrativa como una posibilidad para comprender el fenómeno analizado.

Las narrativas de experimentación que componen la investigación han sido leídas y releídas muchas veces en la búsqueda de comprender el significado de las experiencias vividas por los estudiantes de pregrado e investigamos a los profesores al escribir nuestros aprendizajes relacionados con nuestra práctica educativa y conocimiento químico. Destaqué los sentidos en las narraciones cuando noté un cambio visible en el significado de la situación experimentada y narrada por el sujeto. En el ejercicio de la lectura sucesiva de las narraciones, destaqué los significados de la experiencia vivida. Los significados resaltados no eran rígidos, sino que estaban relacionados con las experiencias vividas en el proceso de investigación.

En la investigación narrativa, las personas viven vidas históricas en paisajes históricos. No es tanto decir que las personas, los lugares y las cosas son de una manera u otra, sino que tienen una historia, un texto cambiante (Clandinin; Connelly, 2011). Por lo tanto, se plantea un dilema cuando el análisis fenomenológico propone unidades de significado como frases que indican momentos distinguibles en todo el texto (Bicudo, 2011). No hay forma de fragmentar vidas históricas, la narrativa involucra una trama y un escenario que juntos crean la calidad experiencial de la narrativa que conforma el paisaje narrado.

Son historias de los temas que el investigador narrativo entiende e interpreta. En la investigación narrativa, compusimos nuestras propias narrativas, que surgen de vivir, contar, volver a contar y revivir nuestras experiencias, lo que nos ayuda a comprender los textos de investigación que escribimos sobre nuestra experiencia en un contexto dado (Clandinin; Connelly, 2011).

Entendemos que el enfoque fenomenológico-hermenéutico señala un camino teórico y metodológico relevante para comprender la experiencia narrada. En la investigación narrativa, "vale la pena reconocer que la relación entre investigador e investigado tiene lugar en la dimensión de su experiencia, trascendiendo así los roles asignados a estos temas en la investigación científica tradicional” (Dutra, 2002, p.375).

En este sentido, la experiencia hermenéutica de comprender los fenómenos vividos a partir de un diálogo con Gadamer (1999) para componer una interpretación de las mónadas narradas. $\mathrm{El}$ autor informa tres relaciones yo/tú que pueden estar relacionadas con el texto. El primero, el I trata al otro como un objeto. El intérprete toma la posición de un observador ideal y busca leer solo lo que dice el texto. La segunda relación reconoce al otro como persona, pero afirma conocer al otro desde el punto de vista de él, el intérprete. La tercera relación Yo/Tú realmente te experimenta como un Tú, es decir, sin ignorar su reclamo, dejando que realmente nos diga algo. En esta tercera relación, el intérprete debe escuchar el texto, para que el texto hable como otra persona en diálogo con usted (Gadamer, 1999). 
Por lo tanto, entendemos que, en la investigación narrativa, las historias contadas y escritas no se refieren necesariamente a un momento y lugar específicos de la investigación, ni a un número exacto de sujetos entrevistados o narraciones escritas. Cuando la experiencia de los sujetos es el foco de la investigación, el investigador también revive sus experiencias y recuerdos de otro tiempo, de otro lugar, y por lo tanto se traen y narran otras historias. Por lo tanto, se planteó un desafío: ¿cómo explicar y narrar el proceso de interpretación narrativa de una manera narrativa? El encuentro con las mónadas en las obras de Walter Benjamin, la reinterpretación del texto "O Narrador" con una mirada atenta a la forma de escribir del autor, el estudio y la comprensión sobre el uso de las mónadas fueron importantes para construir la contribución teórica y metodológica de investigación.

La disposición de las mónadas producirá efectos de comprensión en los lectores dentro de un espectro de posibles regímenes de verdad para su contexto. Las narrativas que componen articulo son formas de decir acerca de nuestras experiencias y se expresan mediante un conjunto de mónadas, que son chispas de significados que hacen que las narrativas sean más que comunicables: las hacen experimentables (Rosa et al, 2011). Las mónadas son una forma de componer significado, lo que permite la construcción de interpretaciones y argumentos sobre la investigación narrativa y la experimentación en la formación de profesores de química.

El encuentro con el concepto de mónada se produjo a partir de la investigación de Rosa et al (2011), quien presenta a las mónadas como otra forma de diseño curricular, a través de la construcción de un currículo narrativo, en el que el aprendizaje narrativo puede verse como central para comprender y establecer una forma diferente de aprendizaje y determinar cómo se puede hacer ese aprendizaje (Rosa et al, 2011).

Para los autores, las mónadas son parte-todo, no partes de un todo. Pueden ser un conjunto de extractos de las transcripciones de las entrevistas, expresadas como breves crónicas, historias o miniaturas de significado que, juntas, narran la coyuntura de un tiempo y un lugar. Afirman que "las narraciones no se clasificarán, porque cualquier tipo de clasificación fijaría verdades, borraría las ambigüedades contenidas en las historias y reduciría en gran medida el espectro de resignificación de las experiencias vividas” (Rosa et al, 2011, p.154).

Las mónadas presentadas en este artículo fueron narradas por profesores e investigadores, y también por estudiantes de Química. Son mónadas escritas en ruedas de formación y otras documentadas en diarios guardados, ya que es un espacio de investigación constante por parte del colectivo docente universitario. Expresan el aprendizaje narrativo, el conocimiento y las experiencias pedagógicas basadas en el desarrollo de la experimentación en química en las ruedas de investigación narrativa. Por lo tanto, presentamos los entendimientos sobre la investigación narrativa en la formación de maestros de Química, para esto establecemos una relación entre Yo/Nosotros al escuchar atentamente lo que se dice, lo que se narra en cada mónada.

\section{EL PAPEL DE LA EXPERIENCIA NARRATIVA EN LA FORMACIÓN DE PROFESORES DE QUÍMICA}

Entendemos la narrativa como una forma de construir conocimiento a través de las experiencias que nos suceden en la formación de maestros de Química. Por lo tanto, presentamos algunos 
entendimientos sobre el papel de la experiencia narrativa en cada mónada, en paralelo construimos un diálogo con Larrosa (2011) y Contreras (2013) que proponen investigación educativa desde la perspectiva de la experiencia desde el proceso de pensamiento. Según estos autores, el acto de pensar favorece algo nos ocurre, nos afecta.

La formación del profesorado se considera como una experiencia cuando uno percibe los acontecimientos vividos y el significado para quienes los viven, pero para eso es necesario detenerse para mirar, para pensar lo que hace lo vivido en usted (Contreras, 2013). En la mónada narrada por la profesora Eva notamos este momento de pausa:

Como me conozco como estudiante y luego como profesional, siempre me he encontrado en el mismo lenguaje de adoctrinamiento. Al principio, la formación católica, luego dentro de sí misma, la formación de contenidos científicos a expensas del conocimiento filosófico, sociológico e histórico, me hizo, al reflejar a un religioso, elegir Química, lo que parecía hacerme escapar del adoctrinamiento. Pero, me doy cuenta de que caí en otro adoctrinamiento, el de la objetividad, las certezas, las verdades. Por supuesto, aprendí mucho porque estudié para enseñar, pero se moldea para enseñar verdades sobre el átomo, sus modelos que ni siquiera entendí, pero que expliqué, sin mostrar dudas y, sin comprender el modelo atómico de Schöerendiger, un poco más de cinética y equilibrio, termodinámica por lejos y cuántos otros podría nombrar. Estoy seguro de que en el momento en que me di cuenta de lo poco que sabía fue cuando elegí Educación y me concentré en analizar mi clase en el doctorado. A mi regreso, esto tuvo implicaciones importantes para el plan de estudios del curso de Grado Químico en FURG, en el que trajo la idea de la escritura y la investigación como elementos estructurantes del aprendizaje. Eso fue alrededor de la década de 2000 (Diario de la profesora Eva).

Los recuerdos de un estudiante en una escuela católica son narrados por Eva, una formación inicial adoctrinada por el contenido científico, en detrimento de otros contenidos como el filosófico y el histórico, lo que lleva a la elección del curso de Química. En la graduación de Química, el encuentro con otro adoctrinamiento, el de la objetividad, las certezas, las verdades. Estudió para enseñar, enseñar verdades sobre el átomo y sus modelos. En el doctorado, al analizar su clase, se dio cuenta de lo poco que sabía. Y así, ideas como la escritura y la investigación se han convertido en elementos estructurantes en la organización de sus clases para intensificar el aprendizaje en química.

El conocimiento de la experiencia conduce a la transformación y reconstrucción de la identidad profesional de ser profesor de química. Contreras (2013) afirma que es necesario recuperar el sentido del conocimiento de la experiencia y su relación con el conocimiento científico y académico, ya que existe una ruptura entre ambas. Él dice que no hay una forma auténtica de saber si saber no es una experiencia, algo que nos transforma. Eva narra esta transformación cuando dice que: [...] estoy segura de que en el momento en que me di cuenta de lo poco que sabía fue cuando elegí Educación y me concentré en analizar mi clase en el doctorado. Rescata el conocimiento de la experiencia educativa como una apertura a otro conocimiento, reconoce sus limitaciones, contradicciones y lagunas.

El enfoque en la escritura narrativa también se retrata en la experiencia de Eva, una maestra que se constituye y transforma a través de la escritura a lo largo de los años. 


\section{E X P E R I E N C I A S}

Tuve algunas experiencias narrativas en las que comencé a buscar la experiencia de los estudiantes a través de la narrativa. Una de ellas en la disciplina de Química Orgánica en la que les pedí a los estudiantes que imaginaran una historia si fueran una molécula de alguna sustancia orgánica. Otra experiencia narrativa que hicimos en la Etapa I fue escribir sobre maestros inolvidables. Aquí, siempre hay muchos informes de maestros de secundaria, como el maestro que salió con el pelo blanco sucio con tiza, o la hermosa maestra de una escuela repleta, así fue como los maestros y profesores perfilaron de alguna manera que marcó a nuestros graduados. Hice este ejercicio durante años, pero me di cuenta de que las marcas eran afectivas, la nota, el cariño, la belleza, es decir, la idea del maestro educador que me di cuenta más tarde después de haber contado algunas de estas historias que todos tenemos. que era necesario buscar el profesor formador inolvidable (Diario de la profesora Eva).

Las experiencias narrativas en el curso de Química son contadas por la profesora Eva, quien recuerda la escritura inolvidable del maestro solicitada en la Práctica pedagógica supervisada I, las marcas afectivas contadas por los académicos, reflejan la importancia de narrar la experiencia vivida con un maestro capacitado inolvidable. La mónada narrada por Eva es una invitación para que su colega, la profesora Ana, encuentre sus escritos del año 2006, conservados hasta hoy por ella. Narra los recuerdos de mi inolvidable maestra de química en la escuela secundaria y refleja la importancia de esta experiencia para su elección profesional.

Mi escuela secundaria fue muy buena, incluso marcada por cambios, otra escuela, una nueva fase en la que aumentaron las responsabilidades. Cuando comenzamos la escuela secundaria comenzamos a pensar en lo que haremos después de terminar, qué camino tomar. Hubo muchos descubrimientos, fui un estudiante muy dedicado, estudié mucho, tuve dificultades y en algunas materias no tuve buenos resultados. Recuerdo que mis mejores notas siempre estuvieron en la disciplina de Química. Admiré y alabé las clases de química. Lo que me hizo gustar tanto la Química en ese momento fue porque creo que es maravilloso saber que todo lo que comemos y usamos contiene Química. Otra razón fue mi maestra de química de secundaria, creo que con ella adquirí mi gusto por la química. Recuerdo que sus clases no fueron agotadoras, aunque la escuela no proporcionó un laboratorio de ciencias, pero ella diseñó experimentos en el aula, fueron maravillosos. Guardo muchos recuerdos, y quiero llevar la experiencia a mis futuras clases de la forma en que manejó sus clases, una maestra seria, pero tenía la amistad de todos los estudiantes, trató a todos por igual y nos transmitió que su responsabilidad era enseñar y la nuestra. para aprender, ella siempre estaba lista para ayudar, por supuesto para aquellos que mostraban interés. Esos momentos de nuestra vida ciertamente no se olvidan, si para algunos solo queda el recuerdo del maestro que realizó las pruebas difíciles, para mí quedaron los buenos recuerdos, porque mientras más conociamiento e información obtengamos hoy, mejor para nosotros en el futuro (Diario del profesora Ana).

Percibimos el proceso de transformación a partir de las experiencias narrativas vividas en cada mónada, y el paso del tiempo aquí no es cronológico, porque lo importante es rescatar estos recuerdos que se convierten en experiencias de formación a través de la escritura narrativa.

Queremos hablar sobre el papel de la experiencia narrativa en la formación del profesorado, pero específicamente en el Grado en Química que busca documentar las experiencias formativas, experiencias narrativas del profesor inolvidable que de alguna manera produce afecto, deja marcas , huellas en la decisión de enseñar. Conviértete en un sujeto de experiencia, un sujeto de 
formación y transformación. Según Larrosa (2002, p.24) “el tema de la experiencia sería algo así como un territorio de paso, algo así como una superficie sensible que lo que sucede afecta de alguna manera. El tema de la experiencia es, sobre todo, un espacio donde tienen lugar los eventos”.

Las mónadas narradas hasta ahora cuentan la historia basada en la formación inicial de los docentes, la historia construida con los demás, con las experiencias vividas colectivamente, lo que favorece el entrelazamiento de ideas y el fortalecimiento de la formación de la identidad profesional de ser un profesor de química. Las mónadas también cuentan la apuesta de las actividades experimentales vividas como experiencia por docentes y académicos en las reuniones semanales del componente curricular:

En las reuniones semanales, se leían textos, la conversación sobre ir a la escuela y, durante el semestre en cada clase, los entrenadores discutían las actividades experimentales propuestas. Recuerdo los momentos de discusión de las actividades experimentales, porque existía el temor de explicar un concepto incorrecto y, la mayoría de las veces, los estudiantes de la Rueda no comentaban sobre la actividad, y la discusión se centró en los maestros que realizaron problematización del conocimiento químico. (Diario del profesora Ana).

Los estudiantes narran en sus diarios como es importante incluir la discusión sobre la experimentación en Química en la escuela. Pero algunos recuerdos permanecen y muestran los desafíos enfrentados y la forma en que solucionamos algunos impasses. Uno de los recuerdos es el de los estudiantes silenciosos, es decir, cuando se les pregunta algo y no constestan. Otro cuya interpretación sigue la misma línea es el interrogatorio de uno de los maestros que hace que un estudiante salga por la puerta. Nuestro enfoque, como docentes, era llamar la atención sobre el error, porque en la lógica de ese momento fue así como entendimos nuestro papel problemático, pero a los estudiantes no les gustó nada (Diario de la profesora Eva).

Las narrativas de los estudiantes se refieren al miedo a explicar o cuestionar el conocimiento químico, pero al mismo tiempo narran las posibilidades de crear y desarrollar una actividad experimental y, por lo tanto, vivir la experiencia docente en la escuela. La profesora Eva también revivió la experiencia y narra el gusto por la disciplina y los desafíos enfrentados, los impases con los estudiantes silenciosos y la reflexión sobre la problematización, con la idea de llamar la atención sobre el error conceptual.

Los profesores de química durante su formación académica desarrollan varios experimentos, principalmente en disciplinas específicas. En Química, la idea de hacer un experimento es común, el laboratorio es un lugar de encanto, es un lugar para pruebas, verdades y repeticiones de técnicas para alcanzar resultados pronosticados. Sin embargo, la formación de un profesor de Química necesita unir el lenguaje técnico y científico con la experiencia vivida por los sujetos que llegan allí para convertirse en maestros. El experimento y la técnica también puede ser los mismos, pero la experiencia de investigarlos en cada una de sus ocurrencias es diferente y única. La experiencia, por lo tanto, siempre tiene algo nuevo, algo sorprendente (Larrosa, 2011).

Por esta razón, durante algunos años, la experimentación en química se propone como un experimento y, por lo tanto, las ideas se reconstruyen y se transforman como la narración en la mónada: 
En el primer semestre de 2014 y 2015, los martes por la mañana, en rueda, nosotros, los maestros de la disciplina y los estudiantes de pregrado, llevamos a cabo el proceso de investigación de ser un profesor de Química a través de la experimentación, el cuestionamiento y la escritura narrativa. El proceso de narrar lo que me sucedió y aprendí de la experimentación fue una experiencia de aprendizaje em la rueda; Algunos describían los materiales, procedimientos y resultados del experimento; otros una breve narrativa de lo sucedido. En reuniones semanales leímos y hablamos sobre lo que senarraba,y asícontinuó el proceso de investigación, la narración nos provocó a repensar, mejorar y reconstruir el conocimiento químico y la constitución de ser profesor de Química. Junto con los estudiantes universitarios estamos logrando formar un colectivo que aprende uno com el outro mediante las preguntas de la rueda. Las preguntas no solo fueron formuladas por nosotros, los maestros, sino también por los estudiantes de pregrado, por lo que todos allí sintieron que eran aprendices al investigar la experimentación propuesta. Al comienzo de las clases, hablamos sobre entendernos a nosotros mismos como maestros que estamos aprendiendo juntos sobre la experimentación. (Diario del profesora Aline)

La experiencia, cuando se entiende como lo que nos sucede deja de ser visto solo como la realización de una técnica de "hacer experimentos” y la prueba de teorías. Se convierte en la experiencia del sujeto, que es un paso hacia la incertidumbre, el cuestionamiento y el aprendizaje durante el curso de la capacitación. Larrosa (2011) la experiencia es un acontecimiento, no se puede causar, no se puede anticipar como un efecto de sus causas. El acontecimiento escapa al orden de causas y efectos"(p.14).

Así, la técnica no es la más importante, pero la experiencia de los sujetos en este proceso de experimentación, sus preguntas, conocimientos iniciales e incertidumbres, en este sentido, afirmamos la importancia de concebir la experimentación como un evento en las clases de Química y en la formación del profesorado. Larrosa (2011) dirá que es necesario “descontaminar” la palabra "experiencia” de las advertencias empíricas y empiristas que se han incorporado en los últimos siglos. La experiencia no es un experimento en el camino de las ciencias experimentales, la experiencia es subjetividad y transformación.

La palabra experimento solo dirige la técnica, ya que la experimentación trae consigo la idea de acción, entonces defendemos la experimentación en química siendo convertida en una experiencia en las dimensiones señaladas por el autor de subjetividad y transformación, siempre que se realice con intención de provocar preguntas durante su desarrollo.

Nuestras apuestas están en línea con lo que dice Larrosa (2011) no es posible saber de antemano cuál será el resultado de un experimento, a dónde nos puede llevar, qué nos hará. Esto se debe a que la experiencia no se trata de planificación lineal, tiempo de pronóstico. La incertidumbre es constitutiva para él, por eso la experiencia siempre supone una apuesta por lo que no se conoce”.

Argumentamos que la experimentación en química como una experiencia es una invitación a proponer autoría, invención, investigación. Por lo tanto, no es necesario que una actividad experimental sea probada y repetida en numerosas ocasiones, por el contrario, queremos que las actividades experimentales se conviertan en eventos en la formación de maestros, para esto es esencial narrar nuestras experiencias sobre el fenómeno investigado, las incertidumbres e las preguntas. 


\section{CONSIDERACIONES FINALES}

La investigación narrativa realizada favoreció la construcción de aprendizajes por parte de los autores (docentes del componente curricular) al narrar sus vivencias en relación a sus prácticas pedagógicas, así como la oportunidad de promover una formación horizontal con los estudiantes, y vivir la experiencia narrativa en la enseñanza de la Química.

Buscamos documentar nuestros aprendizajes del papel de la experiencia narrativa en las ruedas de investigación narrativa en la formación de los profesores de Química como una posibilidad para construir conocimientos, compartir experiencias y, así, favorecer la apropiación del lenguaje narrativa en Química. Desde nuestras propias narrativas como docentes, reforzamos la importancia de la investigación educativa desde la perspectiva de la experiencia. Así, las narrativas documentan una historia de formación, se reviven y se vuelven a contar como una forma de reforzar el papel de la experiencia narrativa en el desarrollo de prácticas pedagógicas y en la constitución de la identidad profesional de los profesores de Química.

Las ruedas de la investigación narrativa en la formación de profesores de química permiten vivir y revivir recuerdos, en los que el paso del tiempo no es cronológico, sino reflexivo y transformado en experiencias pedagógicas y educativas a través de la escritura. Discutimos la experiencia narrativa en Química, como una forma de reforzar la apuesta por la escritura como una forma de constituir un maestro y construir conocimiento.

\section{REFERENCIAS}

Bicudo, M.A. (2011) A pesquisa qualitativa olhada para além dos seus procedimentos. In: Bicudo, M.A. (org.) Pesquisa qualitativa segundo a visão fenomenológica. São Paulo: Cortez.

Clandinin,J.; Connelly, M. (2011) Pesquisa Narrativa: experiência e história de pesquisa qualitativa. Tradução: Grupo de Pesquisa Narrativa e Educação de Professores ILEEL/UFU. Uberlândia: EDUFU.

Contreras, J. (2013). Investigar la experiencia educativa. Barcelona: Morata.

Contreras, J. (2014) Prólogo. In: Skliar, C.; Larrosa, J. Experiencia y alteridad en educación. Rosario: Homo Sapiens Ediciones.

Dutra, E. (2002) A narrativa como uma técnica de pesquisa fenomenológica. Estudos de Psicologia, 7(2), 371-378. Disponible: http://www.scielo.br/pdf/epsic/v7n2/a18v07n2.pdf

Dorneles, A. (2016). Rodas de Investigação Narrativa na Formação de Professores de Química: pontos bordados na partilha de experiências. Tese de doutorado. Programa de Pós-Graduação em Educação em Ciências: Química da Vida e Saúde, Universidade Federal do Rio Grande, Rio Grande. Disponible: https://sistemas.furg.br/sistemas/sab/arquivos/bdtd/0000011204.pdf

Gadamer, H.G. (1999) Verdade e método: traços fundamentais de uma hermenêutica filosófica. Tradução e Revisão: Meurer, F.; Giachini, E. Petrópolis, RJ: Vozes.

Geraldi, J. (2010). A aula como acontecimento. São Carlos: Pedro e João Editores.

Larrosa, J. (2002) Notas sobre a experiência e o saber da experiência. Revista Brasileira de Educação, janabr, n.19, 20-28. 


\section{E X P E R I E N C I A S}

Larrosa, J. (2011) Experiência e Alteridade em Educação. Revista Reflexão e Ação, Santa Cruz do Sul, v.19, n.2, 04-27.

Rosa, M.I. (2011) Narrativas e mônadas: potencialidades para uma outra compreensão de currículo. Currículo sem Fronteiras, v.11, n.1, 198-217. Disponible: http://www.curriculosemfronteiras.org/ vol11isslarticles/rosa-ramos-correa-junior.pdf

Suárez, D.H. (2011) Indagación pedagógica del mundo escolar y formación docente. In: Revista del iice, n. 30., 17-32.

Wells, G. (2001) Indagación dialógica: hacia una teoría y una práctica socioculturales de la educación. Barcelona: Paidós. 